\title{
Squeezed-state generation for two-level atoms in a spatially varying field mode
}

\author{
Min Xiao and H. J. Kimble \\ Department of Physics, University of Texas at Austin, Austin, Texas 78712 \\ H. J. Carmichael \\ Department of Physics, University of Arkansas, Fayetteville, Arkansas 72701
}

Received May 1, 1987; accepted June 12, 1987

\begin{abstract}
A spatially varying field mode is included in calculating the squeezing effect for a system of two-level atoms in the good-cavity limit. Two examples of a Gaussian mode field in a ring cavity and a plane-wave field in a standing-wave interferometer are used to demonstrate the quite general method. In qualitative terms, the squeezing predicted for plane waves is preserved. However, for a given value of atomic cooperativity parameter $C$, there is a degradation in squeezing because of the spatially varying field structure.
\end{abstract}

Squeezed-state generation in an atomic vapor interacting with a cavity field mode has been intensively discussed both experimentally ${ }^{1-3}$ and theoretically ${ }^{1,4-8}$ in recent years. Some experiments ${ }^{1,2}$ have been quite successful in observing squeezing effects at a respectable level. Most theories describing these systems take the cavity field as a plane wave for simplicity. This makes it hard for experiments to be compared quantitatively with the theory. In this paper we illustrate how the spatially varying field mode alters squeezing in a simple system consisting of two-level atoms interacting with a single mode of a high-finesse cavity. We use the general formulas developed earlier in Ref. 9. Two examples, a Gaussian mode in a ring cavity and a plane wave in a standing-wave cavity, are given to illustrate the effect of field variations. Figures and discussions are given for each case and compared with the existing plane-wave theory. ${ }^{5}$

Following Ref. 9, we consider a single, quantized, spatially varying cavity mode, interacting with a collection of $N$ homogeneously broadened two-level atoms that are driven by a coherent classical field of amplitude $\epsilon$. The master equation for the density operator $\hat{\rho}$ of the atom-field system following from the Hamiltonian in the electric-dipole, rotating-wave and Markovian approximations can be written as ${ }^{10-12}$

$$
\begin{aligned}
& \frac{\partial \hat{\rho}}{\partial t}=\frac{1}{i \hbar}\left\{\hbar \omega_{c}\left[\hat{a}^{\dagger} \hat{a}, \hat{\rho}\right]+\frac{1}{2} \hbar \omega_{a} \sum_{\mu=1}^{N}\left[\hat{\sigma}_{\mu}{ }^{z}, \hat{\rho}\right]\right\} \\
& +\sum_{\mu=1}^{N}\left\{g_{\mu} \exp \left(-i \mathbf{k} \cdot \mathbf{r}_{\mu}\right)\left[\hat{a}^{\dagger} \hat{\sigma}_{\mu}{ }^{-}, \hat{\rho}\right]-g_{\mu} \exp \left(i \mathbf{k} \cdot \mathbf{r}_{\mu}\right)\right. \\
& \left.\times\left[\hat{a} \hat{\sigma}_{\mu}{ }^{+}, \hat{\rho}\right]\right\}+\sum_{\mu=1}^{N}\left\{\left(1 / 2 \gamma_{\|}\right)\left(\left[\hat{\sigma}_{\mu}{ }^{-} \hat{\rho}, \hat{\sigma}_{\mu}{ }^{+}\right]+\left[\hat{\sigma}_{\mu}{ }^{-}, \hat{\rho} \hat{\sigma}_{\mu}{ }^{+}\right]\right)\right\} \\
& +\left({ }^{1} / 4 \gamma_{P}\right)\left(\left[\hat{\sigma}_{\mu}{ }^{z} \hat{\rho}, \hat{\sigma}_{\mu}{ }^{z}\right]+\left[\hat{\sigma}_{\mu}{ }^{z}, \hat{\rho} \hat{\sigma}_{\mu}{ }^{z}\right]\right)+\kappa\left\{\left[\hat{a} \hat{\rho}, \hat{a}^{\dagger}\right]\right. \\
& \left.+\left[\hat{a}, \hat{\rho} \hat{a}^{\dagger}\right]\right\}+\kappa\left\{\epsilon \exp \left(-i \omega_{I} t\right)\left[\hat{a}^{\dagger}, \hat{\rho}\right]-\epsilon^{*} \exp \left(i \omega_{I} t\right)[\hat{a}, \hat{\rho}]\right\} .
\end{aligned}
$$

The operators $\hat{a}^{\dagger}$ and $\hat{a}$ are the single-mode creation and annihilation operators, while $\hat{\sigma}_{\mu}{ }^{2}$ and $\hat{\sigma}_{\mu}{ }^{ \pm}$are the Pauli atom- ic operators, $\omega_{c}$ is the cavity resonance frequency, $\omega_{a}$ is the atomic resonance frequency, and $\omega_{I}$ is the driving-field frequency. $g_{\mu}$ is the coupling coefficient between the cavity field mode and an atom at position $\mathbf{r}_{\mu}$ and is given in terms of the normalized mode function $U\left(\mathbf{r}_{\mu}\right)$ by ${ }^{13}$

$$
g_{\mu}=\left(\frac{\mu^{2} \omega_{c}}{2 \hbar \epsilon_{0}}\right)^{1 / 2}\left|U\left(\mathbf{r}_{\mu}\right)\right| \equiv g_{0}\left|U\left(\mathbf{r}_{\mu}\right)\right|
$$

$\kappa$ is the cavity damping rate, $\gamma \|$ is the Einstein $A$ coefficient for spontaneous emission, and $\gamma_{p}$ is the rate of collisioninduced phase decay, so that the total rate of decay of the atomic polarization $\gamma_{\perp}$ is given by $\gamma_{\perp}=\gamma_{\| / 2}+\gamma_{p}$. The thermal photon numbers in both the atom and cavity-mode reservoirs are set to zero.

We divide the cavity mode into $M$ small sections, according to the mode structure, so that the field can be viewed as effectively constant across each section. The sections are still assumed to be large enough so that the number of atoms in the $j$ th section $N_{j} \gg 1$ for all $j$. We checked this assumption for realistic experimental situations with different mode structures and found that it can be reasonably well satisfied. Following Refs. 9-11, we transform the operator master equation (1) into a $c$-number generalized FokkerPlanck equation in the positive $P$ representation. ${ }^{11,14,15} \mathrm{Ow}$ ing to the condition $N_{j} \gg 1$ for each section, we can truncate this generalized Fokker-Planck equation to second order and write corresponding Ito stochastic differential equations. ${ }^{16,17}$ For a high-Q cavity $\left(\gamma_{\perp}, \gamma_{\|} \gg k\right)$, we then adiabatically eliminate atomic variables to arrive at equations for field variables alone.

By setting the derivatives to zero and neglecting the fluctuations in the resulting stochastic differential equations for the field, we find that the steady-state solution satisfies

$$
Y=X\left|1+i \phi+\frac{2(1-i \delta)}{1+\delta^{2}} \cdot \frac{C}{s} \sum_{j=1}^{M} \frac{\left|U\left(\mathbf{r}_{j}\right)\right|^{2} \Delta V_{j}}{1+\frac{V_{\text {eff }}}{s} X \frac{\left|U\left(\mathbf{r}_{j}\right)\right|^{2}}{1+\delta^{2}}}\right|^{2} .
$$


$X=x x^{*}$ and $\mathrm{Y}=y y^{*}$ are cavity- and input-field intensities, where $\left(x, x^{*}\right)=\left(\alpha / \sqrt{n_{0}}, \alpha^{*} / \sqrt{n_{0}}\right)$ are the cavity-field amplitude variables at steady state and $\left(y, y^{*}\right)=\left(\epsilon / \sqrt{n_{0}}, \epsilon^{*} / \sqrt{n_{0}}\right)$ are the input-field amplitude variables, normalized by the saturation photon number $n_{0}=\left(\gamma_{\perp} \gamma \| V_{\text {eff }}\right) /\left(4 g_{0}^{2} s\right) . \quad \delta=\left(\omega_{a}-\right.$ $\left.\omega_{I}\right) / \gamma_{\perp}$ is the dimensionless atomic detuning, and $\phi=\left(\omega_{c}-\right.$ $\left.\omega_{I}\right) / \kappa$ is the dimensionless cavity detuning, with $s$ as the ratio of the atomic sample length to the cavity length, the cooperativity parameter $C=\left(g_{0}^{2} \rho s\right) /\left(2 \gamma_{\perp} \kappa\right)$, where $\rho$ is the density of the uniformly distributed atomic sample, and $\Delta V_{j}$ is the volume of the $j$ th section $\left(N_{j}=\rho \Delta V_{j}\right)$. The effective mode volume is written as ${ }^{13}$

$$
V_{\mathrm{eff}}=s^{2} / \iiint_{\bar{V}}|U(\mathbf{r})|^{4} \mathrm{~d}^{3} r
$$

with $\bar{V}$ the interaction volume. We can take the statistical limit $M \rightarrow \infty$ and $\Delta V_{j} \rightarrow 0$, so that the summation in Eq. (3) can be replaced by an integration:

$$
\lim _{\substack{M \rightarrow \infty \\ \Delta V_{j} \rightarrow 0}} \sum_{j=1}^{M} \Delta V_{j}=\iiint_{\bar{V}} \mathrm{~d}^{3} r
$$

Since we are studying fluctuations around the steady state, we linearize the differential equations for field variables by defining $\delta \bar{x} \equiv \bar{x}-x$, and $\delta \bar{x}^{\dagger} \equiv \bar{x}^{\dagger}-x^{*}$. The cavityfield amplitude variables $\left(\bar{x}, \bar{x}^{\dagger}\right)$ are not complex conjugate in the positive $P$ representation, except at steady states when they take values $\left(x, x^{*}\right)$. The linearized field equations read as

$$
\left(\begin{array}{c}
\delta \dot{\bar{x}} \\
\delta \dot{\bar{x}}^{\dagger}
\end{array}\right)=-M_{\mathrm{ss}}\left(\begin{array}{c}
\delta \bar{x} \\
\delta \bar{x} \dagger
\end{array}\right)+\frac{1}{\sqrt{n_{0}}} B\left(\begin{array}{l}
\xi_{1}(\tau) \\
\xi_{2}(\tau)
\end{array}\right)
$$

where $\left\langle\xi_{i}(\tau) \xi_{j}\left(\tau^{\prime}\right)\right\rangle=\delta\left(\tau-\tau^{\prime}\right) \delta_{i j}$ and $B^{T} B=D_{\mathrm{ss}}$, with

$$
M_{\mathrm{ss}} \equiv\left(\begin{array}{ll}
a & b \\
b^{*} & a^{*}
\end{array}\right), \quad D_{\mathrm{ss}} \equiv\left(\begin{array}{ll}
-P_{s s} & Q_{s s} \\
Q_{s s} & -P_{\mathrm{ss}}
\end{array}\right)
$$

The linearized drift and diffusion coefficients are calculated at the steady-state value. With the Eq. (5), we have, from Eqs. (11) and (17) of Ref. 9,

$$
\begin{array}{r}
a=1+i \phi+\frac{2(1-i \delta) C}{s} \iiint_{\bar{V}} \frac{|U(\mathbf{r})|^{2} \mathrm{~d}^{3} r}{1+\delta^{2}+\frac{V_{\mathrm{eff}} X}{s}|U(\mathbf{r})|^{2}} \\
-\frac{2(1-i \delta) X C V_{\mathrm{eff}}}{s^{2}} \iiint_{\bar{V}} \frac{|U(\mathbf{r})|^{4} \mathrm{~d}^{3} r}{\left[1+\delta^{2}+\frac{V_{\mathrm{eff}} X}{s}|U(\mathbf{r})|^{2}\right]^{2}} \\
b=-\frac{2(1-i \delta) x^{2} C V_{\mathrm{eff}}}{s^{2}} \iiint_{\bar{V}} \frac{|U(\mathbf{r})|^{4} \mathrm{~d}^{3} r}{\left[1+\delta^{2}+\frac{V_{\mathrm{eff}} X}{s}|U(\mathbf{r})|^{2}\right]^{2}}
\end{array}
$$

and

$$
\begin{aligned}
P_{\mathrm{ss}}= & \frac{2 C x^{2} V_{\mathrm{eff}}}{s^{2}} \iiint_{\bar{V}} \frac{\mathrm{d}^{3} r|U(\mathbf{r})|^{4}}{\left[1+\delta^{2}+\frac{X V_{\mathrm{eff}}}{s}|U(\mathbf{r})|^{2}\right]^{3}}\left[(1-i \delta)^{3} f\right. \\
& \left.+i \delta(1-i \delta)(1-f) \frac{X V_{\mathrm{eff}}}{s}|U(\mathbf{r})|^{2}+\frac{1}{2} \cdot \frac{X^{2} V_{\mathrm{eff}}^{2}}{s^{2}}|U(\mathbf{r})|^{4}\right] \\
Q_{\mathrm{ss}}= & \frac{2 C X V_{\mathrm{eff}}}{s^{2}} \iiint_{\bar{V}} \frac{\mathrm{d}^{3} r|U(\mathbf{r})|^{4}}{\left[1+\delta^{2}+\frac{X V_{\mathrm{eff}}}{s}|U(\mathbf{r})|^{2}\right]^{3}}\left\{\left(1+\delta^{2}\right)\right. \\
& \times(1-f)+\left[2+(1-f) \delta^{2}\right] \frac{X V_{\mathrm{eff}}}{s}|U(\mathbf{r})|^{2}+\frac{1}{2} \\
& \left.\cdot \frac{X^{2} V_{\mathrm{eff}}^{2}}{s^{2}}|U(\mathbf{r})|^{4}\right\}
\end{aligned}
$$

where $f=\gamma_{\|} / 2 \gamma_{\perp}$ is the measure of the collision damping.

The question of squeezed-state production is addressed by considering the spectrum of squeezing $S^{\text {out }}(\Omega, \theta)$ for the output field transmitted by one of the cavity mirrors with decay constant $\kappa_{1}$. In terms of the intracavity operators

$$
\hat{A}_{\theta}(t)=\left[\hat{a}(t) e^{-i \theta}+\hat{a}^{\dagger}(t) e^{i \theta}\right],
$$

we have

$$
\begin{aligned}
S^{\text {out }}(\Omega, \theta) & =2 \kappa_{1} \int_{-\infty}^{+\infty} \mathrm{d} \tau\left\langle T: \hat{A}_{\theta}(t) \hat{A}_{\theta}(t+\tau):\right\rangle e^{-i \Omega \tau} \\
& =2 \kappa_{1}\left[S_{12}(\Omega)+S_{21}(\Omega)+S_{11}(\Omega) e^{-2 i \theta}+S_{22}(\Omega) e^{2 i \theta}\right],
\end{aligned}
$$

with $T$ denoting time order and the colons normal order. The spectral matrix $S_{i j}$ of amplitude fluctuation of the complex field $\left(\hat{a}, \hat{a}^{\dagger}\right)$ can be calculated from $M_{\mathrm{ss}}$ and $D_{\mathrm{ss}}$ as ${ }^{18,19}$

$$
S(\Omega)=\frac{1}{n_{0}}\left(M_{\mathrm{ss}}+i \Omega\right)^{-1} D_{\mathrm{ss}}\left(M_{\mathrm{ss}}{ }^{T}-i \Omega I\right)^{-1} .
$$

Since squeezing is optimized at a particular frequency $\Omega_{0}$ by the choice of phase

$$
e^{2 i \theta}=-\frac{S_{22}{ }^{*}\left(\Omega_{0}\right)}{\left|S_{22}\left(\Omega_{0}\right)\right|}
$$

we have, with the choice of this phase,

$$
S^{\text {out }}(\Omega)=2 \kappa_{1}\left\{S_{12}(\Omega)+S_{21}(\Omega)-2 \operatorname{Re}\left[\frac{S_{22} *\left(\Omega_{0}\right) S_{22}(\Omega)}{\left|S_{22}\left(\Omega_{0}\right)\right|}\right]\right\} \text {. }
$$

For a given spatial mode $U(\mathbf{r})$, we can calculate $a, b, P_{\mathrm{ss}}$, and $D_{\text {ss }}$ by using Eqs. (8) and (9) and then solve Eq. (12) to find the matrix elements $S_{i j}$ required by Eq. (14). For this system, the best squeezing always occurs at $\Omega_{0}=0$; therefore we set $\Omega=\Omega_{0}=0$ in what follows. We further assume an ideal, single-sided cavity, so that $\kappa_{1}=\kappa$. We now discuss the results for two particular examples.

For our first example we take a Gaussian profile in a ring cavity. We divide the Gaussian beam into $M$ cylindrical shells along the cavity axis, each with radius $r_{j}$ and thickness $\Delta r_{j}$. When the Rayleigh length is much longer than the sample length, as is usually the case, ${ }^{1}$ we can neglect the $z$ 


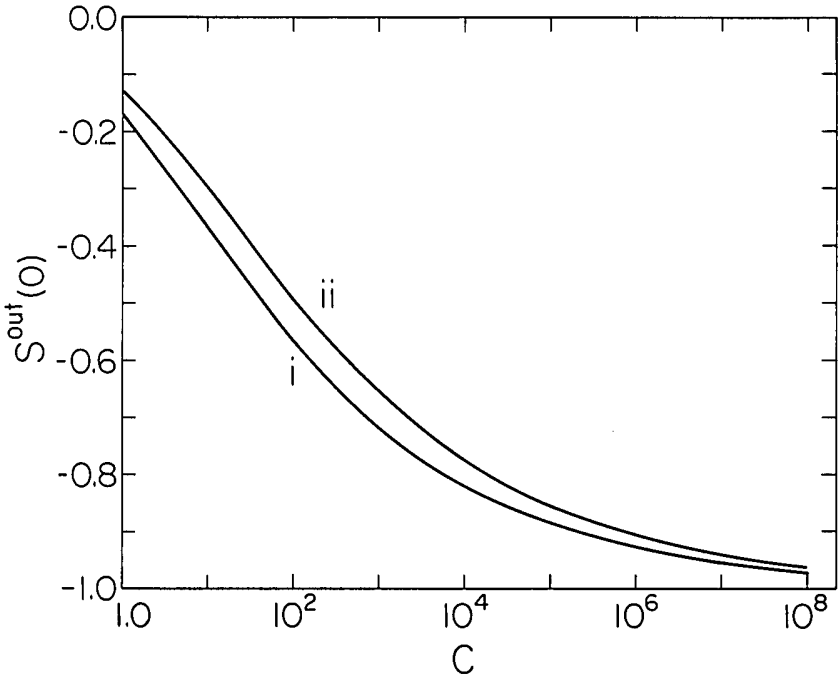

Fig. 1. Optimal squeezing $S^{\text {out }}(0)$ versus atomic cooperativity parameter $C$. Curve i, plane wave in a ring cavity; curve ii, Gaussian mode in a ring cavity. $\delta, \phi$, and $X$ are optimized at each point to give best squeezing. Representative values are as follows: $C=10$ : i, $\delta$ $=7.20, \phi=1.92, X=8.64$; ii $\delta=7.18, \phi=2.10, X=6.92$. $C=10^{2}: \mathrm{i}$, $\delta=28.2, \phi=6.00, X=73.9 ; \mathrm{ii}, \delta=26.5, \phi=6.60, X=54.2 . \quad C=10^{3}$ : i, $\delta=111, \phi=16.7, X=556$; ii, $\delta=101, \phi=18.6, X=388$. $C=10^{4}: \quad \mathrm{i}, \delta=438, \phi=44.2, X=3.87 \times 10^{3} ; \mathrm{ii}, \delta=392, \phi=49.7$, $X=2.64 \times 10^{3} . \quad C=10^{5}: \quad \mathrm{i}, \delta=1.73 \times 10^{3}, \phi=114, X=2.58 \times 10^{4}$; ii, $\delta=1.52 \times 10^{3}, \phi=130, X=1.70 \times 10^{4} . C=10^{6}: \mathrm{i}, \delta=6.86 \times 10^{3}$, $\phi=290, X=1.70 \times 10^{5} ; \mathrm{ii}, \delta=5.72 \times 10^{3}, \phi=348, X=9.97 \times 10^{4}$. $C$ $=10^{7}: \mathrm{i}, \delta=2.73 \times 10^{4}, \phi=731, X=1.11 \times 10^{6} ; \mathrm{ii}, \delta=2.54 \times 10^{4}, \phi$ $=785, X=9.05 \times 10^{5} . C=10^{8}: \quad$ i, $\delta=1.07 \times 10^{5}, \phi=1.87 \times 10^{3}, X$ $=6.76 \times 10^{6} ; \mathrm{ii}, \delta=1.03 \times 10^{5}, \phi=1.94 \times 10^{3}, X=5.90 \times 10^{6}$.

dependence of the beam waist $W$. For the normalized mode function we take

$$
U(\mathbf{r})=\left(\frac{2}{\pi L W^{2}}\right)^{1 / 2} \exp \left(-r^{2} / W^{2}\right),
$$

which with

$$
\mathrm{d}^{3} r=(2 \pi r \mathrm{~d} r) L s
$$

gives from Eq. (4)

$$
V_{\text {eff }}=\left(\pi W^{2} L\right) s .
$$

Substituting from Eqs. (15)-(17) into Eqs. (8) and (9) and computing the integrals, we find the explicit linearized drift and diffusion coefficients

$$
\begin{aligned}
& a=(1+i \phi)+\frac{2 C(1-i \delta)}{1+\delta^{2}+2 X}, \\
& b=\frac{(1-i \delta) C x^{2}}{X}\left[\frac{2}{1+\delta^{2}+2 X}-\frac{1}{X} \ln \left(1+\frac{2 X}{1+\delta^{2}}\right)\right]
\end{aligned}
$$

and

$$
\begin{aligned}
P_{\mathrm{ss}}= & C x^{2}\left(\frac { 1 } { ( 1 + \delta ^ { 2 } + 2 X ) ^ { 2 } } \left\{\frac{2(1-i \delta)^{3} f}{1+\delta^{2}}+4 X+3\left[\delta^{2}(1+2 f)\right.\right.\right. \\
& -2 i \delta(1-f)+3]+\frac{1+\delta^{2}}{X}\left[\left(1+2 f \delta^{2}-2 i \delta(1-f)+3\right]\right\} \\
& \left.-\frac{(1+2 f) \delta^{2}-2 i \delta(1-f)+3}{2 X^{2}} \ln \left(1+\frac{2 X}{1+\delta^{2}}\right)\right)
\end{aligned}
$$

$$
\begin{aligned}
Q_{\mathrm{ss}}= & C\left\{\frac{4 X^{2}-(1+2 f)\left(1-3 \delta^{2}\right) X-\left(1+\delta^{2}\right)\left[1-\delta^{2}(1+2 f)\right]}{\left(1+\delta^{2}+2 X\right)^{2}}\right. \\
& \left.+\frac{1-\delta^{2}(1+2 f)}{2 X} \ln \left(1+\frac{2 X}{1+\delta^{2}}\right)\right\}
\end{aligned}
$$

With Eqs. (18) and (19), we can compute the squeezing effect for a Gaussian mode from Eqs. (7), (12), and (14).

In Fig. $1, S^{\text {out }}(0)$ is plotted against atomic cooperativity $C$, where curve $\mathrm{i}$ is for a plane wave in a ring cavity and curve ii is for a Gaussian mode in a ring cavity. $\delta, \phi$, and $X$ are optimized to give the best squeezing at each point along the curves. It can be seen that squeezing is preserved for the Gaussian profile but degraded relative to the plane-wave case for a given value of $C$. At lower $C$ values $\left(10^{2}-10^{3}\right)$ the discrepancy can be as large as $10 \%$ or more, which is important for a quantitative comparison between experiment and theory. The same degree of squeezing as in the plane-wave case can be recovered for the Gaussian profile only by increasing the atomic cooperativity $C$, the atomic and cavity detunings, and the field intensity. The convergence of the two curves at large $C$ is a consequence of the fact that, in the dispersive limit to which one is driven at large $C$, all effects

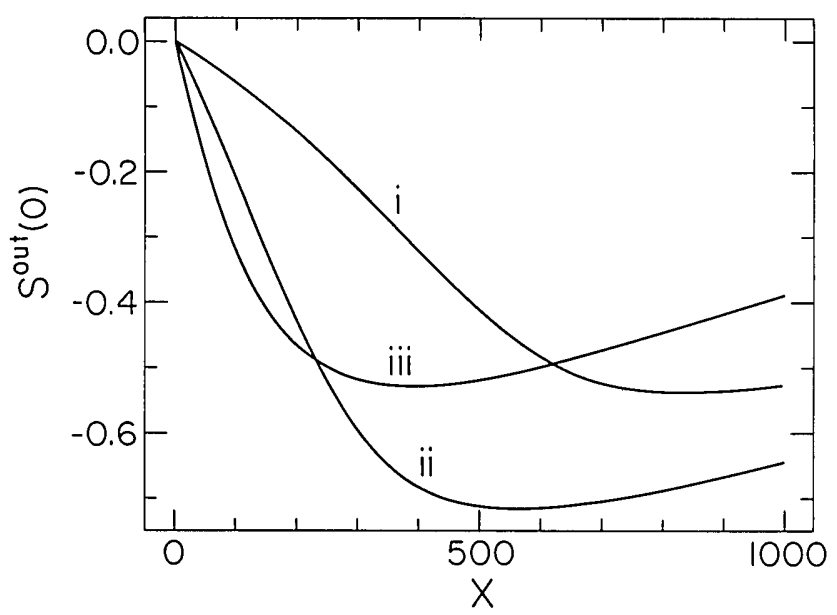

Fig. 2. Squeezing $S^{\text {out }}(0)$ versus intensity $X$ for the plane-wave case. Curve i: $C=10^{3}, \delta=111$ (optimal for given $C$ ), $\phi=15.0$; curve ii: $\mathrm{C}=10^{3}, \delta=111, \phi=16.7$ (optimal); curve iii: $C=10^{3}, \delta=$ $111, \phi=18.0$.

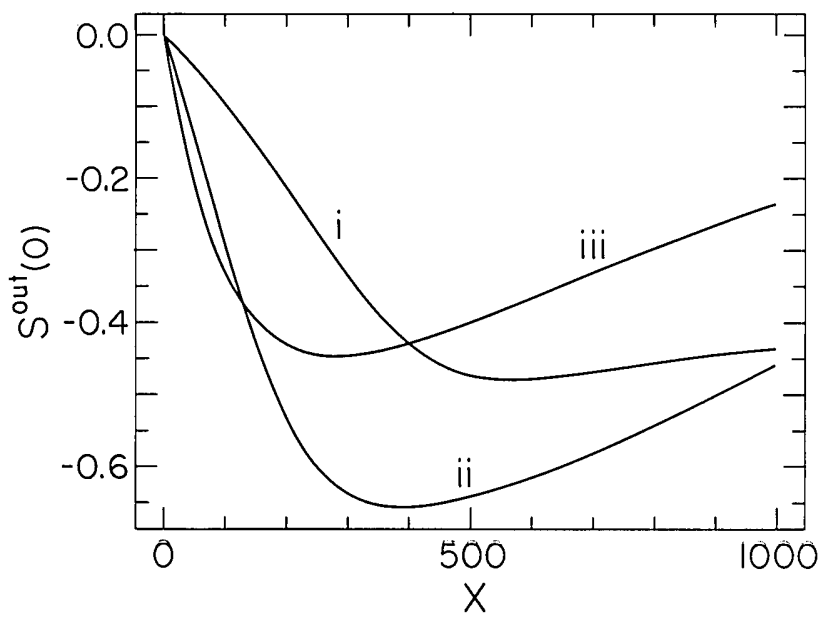

Fig. 3. Squeezing $S^{\text {out }}(0)$ versus intensity $X$ for the Gaussian case. Curve i: $C=10^{3}, \delta=101$ (optimal for given $C$ ), $\phi=17.0$; curve ii: $C=$ $10^{3}, \delta=101, \phi=18.6$ (optimal); curve iii: $C=10^{3}, \delta=101, \phi=20.0$. 
resulting from the spatially varying field mode vanish. 9,13 There are two reasons why we have computed our results out to the high- $C$ limit shown in Fig. 1 . One is to observe the approach to the dispersive limit, and the other is to compare with the results of Reid and Walls. ${ }^{5}$ At $C=2 \times 10^{6}$, the value on curve $\mathbf{i}$ for plane waves is in agreement with their result.

In Figs. 2 and 3 we plot the degree of squeezing $S^{\text {out }}(0)$ versus intensity $X$ for $C=10^{3}$ and for three different values of $\phi$ for plane-wave and Gaussian cases, respectively. $\delta$ is fixed for all three curves to be its optimal value for the given value of $C ; \phi$ is varied slightly around its optimal value. It is clear that the squeezing is very sensitive to cavity detuning. It can also be noticed that the best squeezing in this cross section of the multidimensional parameter space becomes somewhat smaller and more sensitive to the change in intensity for the Gaussian mode structure.

Turning next to the case of a standing-wave cavity with a plane-wave transverse profile, we divide the cavity into regions of length $\lambda / 4$ and these regions into $M$ small sections in the $z$ direction. Each region of length $\lambda / 4$ makes an identical contribution to the sum over atoms. The normalized mode function is

$$
U(\mathbf{r})=\left(\frac{2}{A L}\right)^{1 / 2} \cos (k z)
$$

and

$$
\mathrm{d}^{3} r=A \mathrm{~d} z,
$$

where $A$ is the cross-sectional area of the cavity and $k$ is the wave vector. With Eqs. (20) and (21), Eq. (4) becomes

$$
V_{\text {eff }}=\left(\frac{2}{3} A L\right) s .
$$

Substituting Eqs. (20)-(22) into Eqs. (8) and (9), we have

$$
\begin{aligned}
& a=(1+i \phi)+\frac{2 C(1-i \delta)}{\left(1+\delta^{2}\right)\left[1+\frac{4 X}{3\left(1+\delta^{2}\right)}\right]^{3 / 2}}, \\
& b=\frac{(1-i \delta) 3 C}{x^{* 2}}\left\{\frac{2 X+1+\delta^{2}}{\left(1+\delta^{2}\right)\left[1+\frac{4 X}{3\left(1+\delta^{2}\right)}\right]^{3 / 2}}-1\right\},
\end{aligned}
$$

and

$$
\begin{aligned}
P_{\mathrm{ss}}= & C x^{2}\left\{\frac{(1+2 f)-\delta^{2}\left[4 f+\delta^{2}(1-2 f)\right]-i \delta\left[4 f\left(1-\delta^{2}\right)+2\left(1+\delta^{2}\right)\right]}{\left(1+\delta^{2}\right)^{3}\left[1+\frac{4 X}{3\left(1+\delta^{2}\right)}\right]^{5 / 2}}+\frac{1}{X}+\frac{2\left[1+f \delta^{2}-i \delta(1-f)\right]}{X\left(1+\delta^{2}\right)\left[1+\frac{4 X}{3\left(1+\delta^{2}\right)}\right]^{3 / 2}}\right. \\
& \left.-\frac{3}{X^{2}}\left[1-\frac{1}{\sqrt{1+\frac{4 X}{3\left(1+\delta^{2}\right)}}}\right]\left[\frac{3}{2}+\frac{\delta^{2}}{2}(1+2 f)-i \delta(1-f)\right]\right\} \\
Q_{\mathrm{ss}}= & C\left\{1-\frac{X}{\left.\left(1+\delta^{2}\right)^{2}\left[1+\frac{4 X}{3\left(1+\delta^{2}\right)}\right]^{5 / 2}\left[(1+2 f)+\delta^{2}(1-2 f)\right]-\frac{2\left(1-f \delta^{2}\right)}{4 X}\right]^{3 / 2}}\right. \\
& \left.+\frac{3}{X}\left[1-\frac{1}{\sqrt{1+\frac{4 X}{3\left(1+\delta^{2}\right)}}}\right]\left[\frac{1}{2}-\frac{\delta^{2}}{2}(1-2 f)\right]\right\}
\end{aligned}
$$




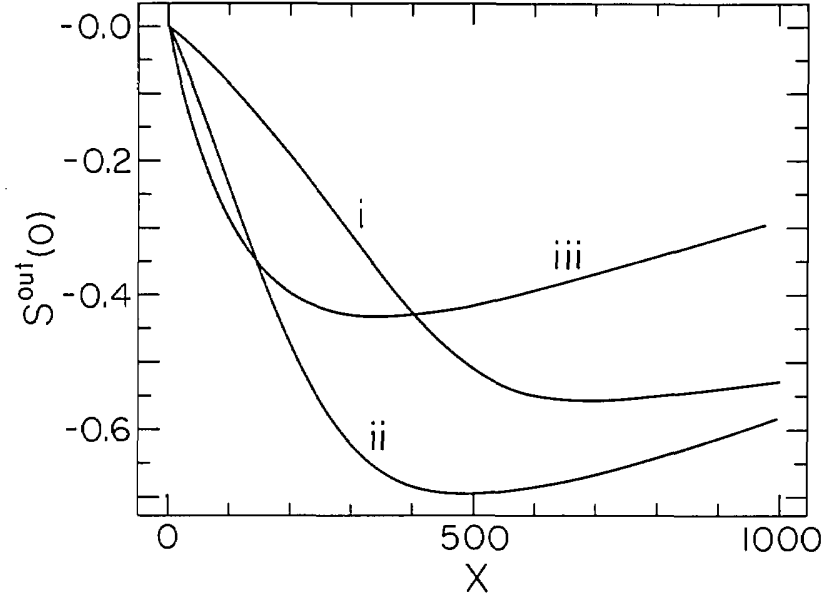

Fig. 5. Squeezing $S^{\text {out }}(0)$ versus intensity $X$ for the standing-wave case. Curve i, $C=10^{3}, \delta=107$ (optimal for given $C$ ), $\phi=16.0$; curve ii, $C=10^{3}, \delta=107, \phi=17.4$ (optimal); curve iii, $C=10^{3}, \delta=107, \phi=$ 19.0 .

small compared with the atomic relaxation rate. Explicit results for two examples, a Gaussian ring interferometer, and a standing-wave interferometer with transverse structure of a plane wave were obtained and compared with existing theory for a plane-wave ring interferometer. Of course, current generations of experiments are far short of achieving the extremely large values of $C$ required to obtain the very large degrees of squeezing shown on the figures. In addition, the nondegenerate case is far more favorable than the degenerate case that we analyzed..$^{1,2,4,7}$ However, we believe that our calculation should indicate that the spatial variations of the intracavity field do have a nonnegligible effect (about 10\%) on the degree of squeezing that can be achieved in realistic experimental situations. The case of a Gaussian mode standing-wave, which is most relevant in current experiments, can also be treated with the formalism developed in Ref. 9 and here.

\section{ACKNOWLEDGMENTS}

This research was supported by the National Science Foundation, by the Venture Research Unit of British Petroleum of North America, and by the U.S. Office of Naval Research.

\section{REFERENCES}

1. M. G. Raizen, L. A. Orozco, M. Xiao, T. L. Boyd, and H. J. Kimble, Phys. Rev. Lett. 59, 198 (1987).

2. R. E. Slusher, L. W. Hollberg, B. Yurke, J. C. Mertz, and J. F. Valley, Phys. Rev. Lett. 55, 2409 (1985).

3. M. W. Maeda, P. Kumar, and J. H. Shapiro, Opt. Lett. 12, 161 (1987).

4. M. D. Reid and D. F. Walls, Phys. Rev. A 31, 1622 (1985); 34, 4929 (1986).

5. M. D. Reid and D. F. Walls, Phys. Rev. A 32, 396 (1985).

6. H. J. Carmichael, Phys. Rev. A 33, 3262 (1985).

7. D. A. Holm and M. Sargent III, Phys. Rev. A 35, 2150 (1987).

8. L. A. Lugiato and G. Strini, Opt. Commun. 41, 67 (1982).

9. M. Xiao, H. J. Kimble, and H. J. Carmichael, Phys. Rev. A 35, 3832 (1987).

10. W. H. Louisell, Quantum Statistical Properties of Radiation (Wiley, New York, 1983).

11. P. D. Drummond and D. F. Walls, Phys. Rev. A 23, 2563 (1981).

12. L. A. Lugiato, in Progress in Optics, E. Wolf, ed. (North-Holland, Amsterdam, 1984), vol. 21.

13. P. D. Drummond, IEEE J. Quantum Electron. QE-17, 301 (1981).

14. H. Haken, Encyclopedia of Physics (Springer-Verlag, Berlin, 1970), Vol. XXV/2C.

15. P.D. Drummond and C. W. Gardiner, J. Phys. A 13, 2353 (1980); P.D. Drummond, C. W. Gardiner, and D. F. Walls, Phys. Rev. A 24, 914 (1981).

16. L. Arnold, Stochastic Differential Equations (Wiley, New York, 1974).

17. H. Risken, The Fokker-Planck Equation (Springer-Verlag, Berlin, 1984).

18. S. Chaturvedi, C. W. Gardiner, I. Matheson, and D. F. Walls, J. Stat. Phys. 17, 469 (1977).

19. C. W. Gardiner, Handbook of Stochastic Processes (SpringerVerlag, New York, 1983).

\section{H. J. Carmichael}

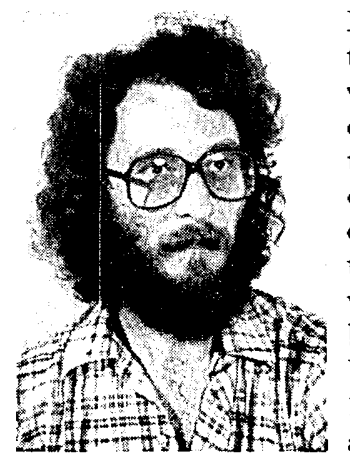

H. J. Carmichael was born in Manchester, England, in 1950. He grew up and was educated in New Zealand. He received his undergraduate education at the University of Auckland, where he received the M. Sc. degree in physics (first class honours) in 1973. In 1977 he obtained the D. Phil. degree from the University of Waikato. He received a Fulbright award in 1977 to travel to the United States, where he remained until 1981 working in postdoctoral positions at City College, City University of New York, and the University of Texas at Austin. After a further year of postdoctoral studies at the University of Waikato, he joined the faculty at the University of Arkansas, Fayetteville, in 1983. His research is in the areas of resonance fluorescence, optical bistability, and optical instabilities. His current interests include the photon statistics of nonclassical optical fields, quantized nonlinear dynamics in optical systems, and intracavity quantum electrodynamics. 